\title{
Unusual Case of Pubertal Gynaecomastia
}

\author{
Sandeep Chaudhary, Anubhav Thukral, Sumit Chakraborty, \\ Satinath Mukhopadhyay, Subhankar Chowdhury
}

Department of Endocrinology, Institute of Post Graduate Medical Education and Research, Kolkata, India

Keywords: puberty, gynaecomastia, mammoplasty

A 13-year-old Indian boy presented with progressive nonpainful enlargement of bilateral breasts for 2 years. (Figure 1) There was no history of any drug intake that could lead to gynaecomastia with no significant past medical history. His BMI was $28.5 \mathrm{~kg} / \mathrm{m}^{2}$, no icterus, no stigmata of liver disease, acanthosis was present, sexual maturity rating P1 $\mathrm{A} 1$, and stretched penile length was $5 \mathrm{~cm}$, testicular volume $3 \mathrm{ml}$ bilaterally of normal consistency; with a bone age of 13 years and a disc diameter of $6 \mathrm{~cm}$ bilaterally. Investigations revealed normal liver function tests, fasting testosterone levels of $67 \mathrm{ng} / \mathrm{dl}$, LH $1.17 \mathrm{U} / \mathrm{L}, \mathrm{FSH} 3.68 \mathrm{U} / \mathrm{L}$, FBS $90 \mathrm{mg} \%$, and PPBS $107 \mathrm{mg} \%$. Considering the overall clinical picture, a diagnosis of Peripubertal Gynaecomastia was made. Obesity could have been a contributing factor to the development of this condition in this patient. He denied having taken any estrogenic compounds but environmental exposure cannot be ruled out as we did not assay for the levels of these environmental endocrine disruptors and estrogenic agents. However, increased pigmentation of nipples and areola is a common feature of gynaecomastia due to exogenous synthetic estrogen exposure which was absent in this case.

Gynaecomastia is defined as the growth of the male mammary gland to become palpable and/or visible. Gynaecomastia is clinically apparent when the diameter of the glandular tissue exceeds $0.5 \mathrm{~cm}$. In early puberty a significant proportion, around $30-60 \%$ of boys, will show some degree of gynaecomastia which regresses spontaneously ${ }^{1}$; while usually bilateral, it can be unilateral in about $20 \%$. Spontaneous regression occurs in the majority of the cases in about 1-2 years, gynaecomastia persists in $<5 \% .{ }^{2}$ However, its presence causes considerable concern for the patients and their parents. Most patients with pubertal or physiological gynaecomastia have disc diameters less than $2 \mathrm{~cm}$ and pathological gynaecomastia is defined as a disc diameter greater than $4 \mathrm{~cm}$ or $2-4 \mathrm{~cm}$ with pain and progressive enlargement. Our case had a disc diameter of $6 \mathrm{~cm}$ but no cause could be ascertained and a diagnosis of pubertal gynaecomastia was entertained.

e-ISSN 2308-118X

Printed in the Philippines

Copyright $@ 2013$ by the JAFES

Received July 23, 2013. Accepted October 9, 2013.
The common causes of pathological gynaecomastia are exogenous estrogen administration, hypergonadotropic hypogonadism, testicular or adrenal tumours secreting estrogen, hCG secreting tumours, aromatase excess syndromes, breast cancer (usually unilateral) and idiopathic. Obesity is also associated with gynaecomastia as increased aromatase activity in the expanded adipose tissue gives rise to more peripheral conversion of testosterone to estrogen. Most cases of pubertal gynaecomastia have P3 pubic hair i.e., they are fairly into mid-puberty, however, our case was in early puberty with possible onset of gynaecomastia prepubertally.

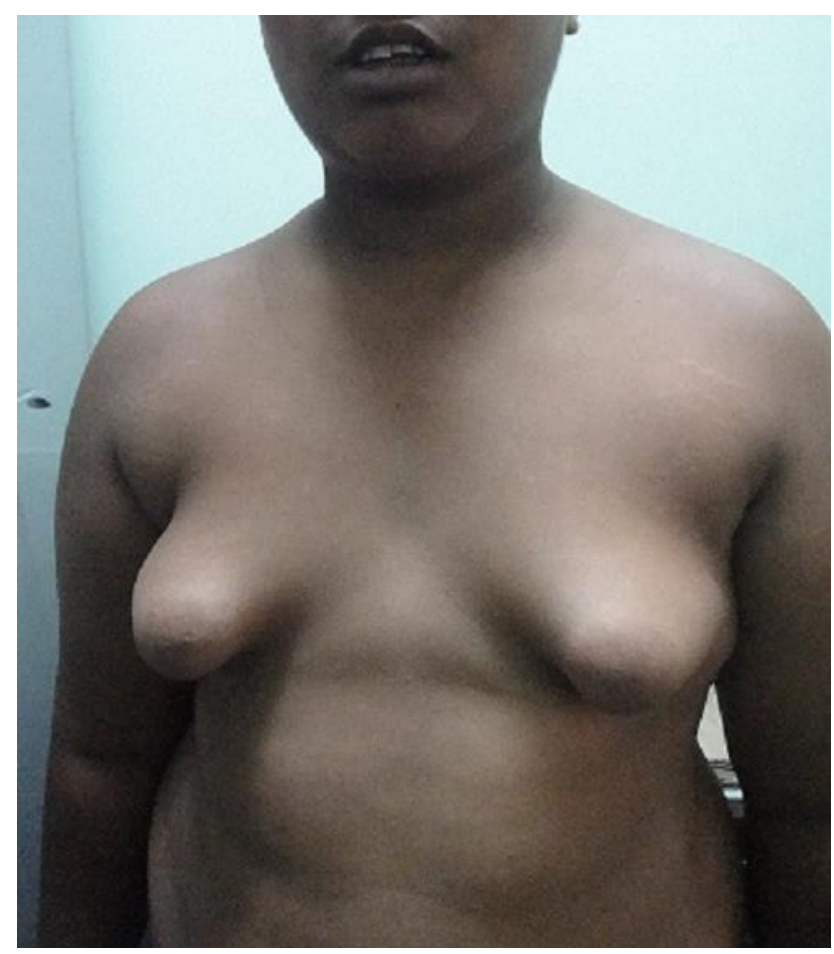

Figure 1. Prominent bilateral gynaecomastia with enlarged areolae and prominent papillae in an obese child

Selective estrogen receptor modulators (SERMS) like tamoxifen and raloxifene have been used in managing gynaecomastia; however they are effective only in the early 6 months, during the painful, proliferative and

Corresponding author: Anubhav Thukral, MBBS, MD

Institute of Post Graduate Medical Education and Research

124/A 418 Block No. 11

Govind Nagar, Kanpur

Uttar Pradesh 208006 India

Tel. No.: $+919903815882 /+919415992944$

E-mail:anubhavthukral@rediffmail.com 
glandular phase of gynaecomastia. Even in this phase response to treatment is seen in reduced pain, reduction in breast size may not be that dramatic. No medical treatment is effective beyond 1 year of gynaecomastia as these are usually non-tender and the breast tissue has become fibrotic and resistant to medical therapy. The only remaining therapy option is surgery. A reduction mammoplasty has been advised for this patient.

\section{References}

1. Ma NS, Geffner ME Gynecomastia in prepubertal and pubertal men Curr Opin Pediatr. 2008

2. Nydick M, et al Gynecomatia in adolescent boys. JAMA 178:449454,1961

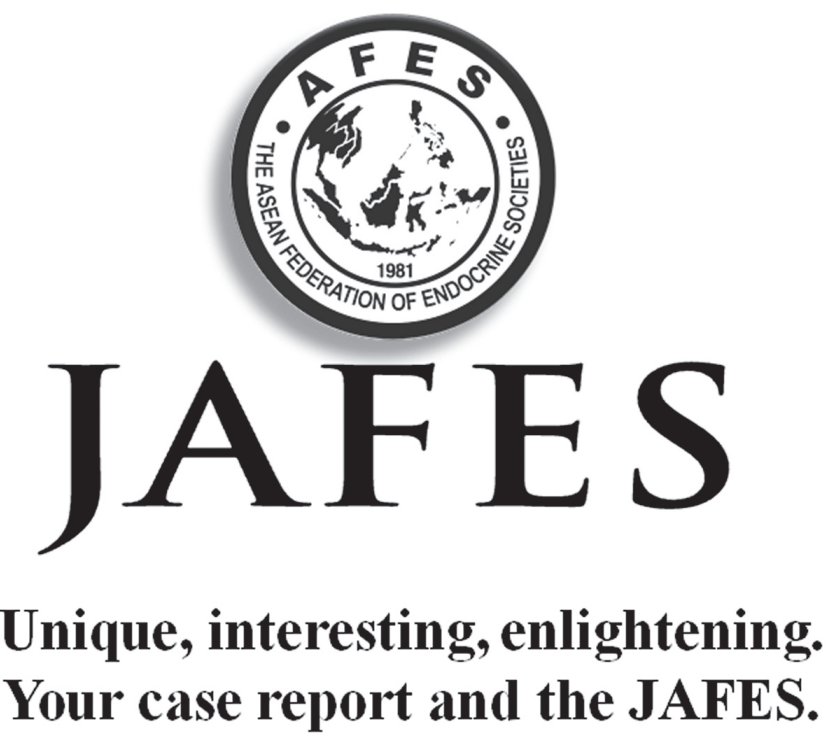

